\title{
Behavioural Neurology
}

\section{Cortical thinning trajectories across disease stages and cognitive impairment in amyotrophic lateral sclerosis}

\author{
Monica Consonni ${ }^{a, 1}$, Eleonora Dalla Bella ${ }^{a, 1}$, Valeria Elisa Contarino ${ }^{b}$, \\ Enrica Bersano ${ }^{a}$ and Giuseppe Lauria ${ }^{a, c, *, 2}$ \\ a 3rd Neurology Unit and Motor Neuron Diseases Center, IRCCS Foundation "Carlo Besta" Neurological Institute, Via \\ Celoria 11, 20133, Milan, Italy \\ b Department of Neuroradiology, Fondazione IRCCS Ca' Granda Ospedale Maggiore Policlinico, Via F. Sforza 28, \\ 20122, Milano, Italy \\ ' Department of Biomedical and Clinical Sciences "Luigi Sacco", University of Milan, Via G.B. Grassi 74, 20157, \\ Milan, Italy
}

\section{A R T I C L E I N F O}

Article history:

Received 25 October 2019

Reviewed 29 December 2019

Revised 12 May 2020

Accepted 16 July 2020

Action editor Brad Dickerson

Published online xxx

Keywords:

Amyotrophic lateral sclerosis

MRI

Cortical thickness

Cognition

Staging

\begin{abstract}
A B S T R A C T
Background: Cortical neuron degenerative process underlying upper motor neuron involvement in amyotrophic lateral sclerosis (ALS) spreads to extra-motor regions as disease progresses. This is associated with cognitive and behavioural worsening in more severe disease stages. However, the clinical variability of ALS patients might reflect different cortical involvement in extra-motor areas.

Objectives: To investigate cortical thinning across disease stages in ALS patients accounting for their cognitive/behavioural impairment.

Methods: Thirty-six ALS patients (17 with cognitive/behavioural impairment, ALSimp) and 26 healthy controls underwent structural 3T magnetic resonance imaging. Cortical thickness was measured with a region-wise approach. The King's Clinical Staging System was used to determine disease stages. The Jonckheere-Terpstra test tested for trends in cortical thinning and cognitive involvement across disease stages.

Results: Significant trends toward cortical atrophy across disease stages were found in bilateral frontal and cingular cortex, left temporal gyrus and right occipital gyrus of ALS patients, consistently with cognitive impairment in phonemic fluency, language, verbal episodic memory and social cognition. Sub-group analyses revealed that ALSimp had specific thinning in the right fronto-temporal insular cortex related to more pronounced cognitive involvement.
\end{abstract}

Abbreviations: ALS, Amyotrophic Lateral Sclerosis; ALSbi, ALS patients with mild behavioural impairment; ALSci, ALS patients with mild dysexecutive cognitive impairment; ALScn, ALS patients with normal cognitive/behavioural profile; ALSimp, ALS patients with ALSci and/or ALSbi; CT, Cortical thickness; FTD, Frontotemporal Dementia; KDS, King's disease stage.

* Corresponding author. Department of Clinical Neuroscience, 3rd Neurology Unit, Fondazione IRCCS Istituto Neurologico Carlo Besta, Via Celoria 11, 20133, Milano, Italy.

E-mail addresses: giuseppe.lauriapinter@istituto-besta.it, giuseppe.lauria@unimi.it (G. Lauria).

1 These authors contributed equally to the manuscript.

2 www.istituto-besta.it https://doi.org/10.1016/j.cortex.2020.07.007

0010-9452/@ 2020 The Author(s). Published by Elsevier Ltd. This is an open access article under the CC BY-NC-ND license (http:// creativecommons.org/licenses/by-nc-nd/4.0/). 
Conclusion: Looking at ALS patients irrespective of their cognitive phenotype, motor and extra-motor cortical involvement is consistent with neuropathological studies of disease dissemination. Segregating patients according to their cognitive status, a distinctive trajectory of cortical thinning emerged for ALSimp patients, suggesting a specific course distinct to that of the classic ALS phenotype.

(C) 2020 The Author(s). Published by Elsevier Ltd. This is an open access article under the CC BY-NC-ND license (http://creativecommons.org/licenses/by-nc-nd/4.0/).

\section{Introduction}

The neurodegenerative process of amyotrophic lateral sclerosis (ALS) is characterized by loss of motor neurons of the primary motor cortex, corticospinal tract, brainstem and spinal cord. However, neuronal susceptibility to ALS is not only confined to motor neurons (Piao et al., 2003) as demonstrated by clinical, pathological and genetic overlap of extra-motor pathology with frontotemporal dementia (van Es et al., 2017). It has been suggested that pathological processes underlying ALS begins in motor neurons and propagates to other regions of the brain in a fashion that is predictable based on proximity and connection by a corticofugal axonal spread (Braak et al., 2013; Brettschneider et al., 2013). The trajectory of the spreading pattern seems to be consistent with the inclusion and dissemination of phosphorylated $43-\mathrm{kDa}$ transactive response DNA-binding protein (pTDP-43) pathology (Brettschneider et al., 2013). This suggested that ALS potentially has a focal onset with subsequent spread along brain connections (Verstraete, Veldink, van den Berg, \& van den Heuvel, 2014). Therefore, the extent of pTDP-43 in the prefrontal neocortex could lead ALS patients to eventually develop "frontal type" cognitive deficits (i.e.,: executive and social), depending on disease duration and rapidity of propagation (Brettschneider et al., 2013). Longitudinal neuroimaging studies documented significant grey matter changes over time in motor and extra-motor pathways (Agosta et al., 2009; Chipika, Finegan, Li Hi Shing, Hardiman, \& Bede, 2019; de Albuquerque et al., 2017; R. A. Menke et al., 2014; Schuster et al., 2014; Senda et al., 2011; Verstraete et al., 2012) with, however, several contradictory findings (Table S1). Indeed, decline in cognitive functions has been found to be faster in ALS patients cognitively impaired at diagnosis, whereas patients with normal cognition tended to remain unimpaired (Elamin et al., 2013).

This variability is likely to reflect different anatomical changes. Our in-vivo analysis of cortical thickness (CT) aimed at addressing this issue through the analysis of cortical thinning staging in a well-defined cohort of ALS patients including subgroups differing for cognitive and/or behavioural involvement.

\section{Methods}

\subsection{Participants}

We recruited 40 consecutive patients diagnosed with ALS (Brooks, Miller, Swash, Munsat, \& Diseases, 2000) not carrying mutations in C9orf72, SOD1, FUS or TARDBP genes and 26 healthy control (HC) subjects. Exclusion criteria were comorbid frontotemporal dementia (Rascovsky et al., 2011), Alzheimer's disease (NIA-AA), evidence of another neurologic condition affecting cognition (e.g., head trauma, hydrocephalus, vascular disease), drug or alcohol abuse, neurocognitive developmental disorder, primary psychiatric disorders (e.g., bipolar disorder, major depression), other severe medical conditions and mother tongue other than Italian. Four patients meeting the inclusion criteria were subsequently excluded because of claustrophobia $(n=1)$ and excessive movement artefacts at magnetic resonance imaging (MRI) $\operatorname{scan}(\mathrm{n}=3)$.

\subsection{Materials}

Disability was scored by the revised ALS Functional Rating Scale (ALSFRS-R). Disease duration was calculated from symptom onset to MRI date in months. Clinical stage was estimated in each patient, during neurological examination, using the King's Staging Criteria (Balendra et al., 2014; Roche et al., 2012). Accordingly, 11 ALS patients were at King's disease stage $1(\mathrm{KDS}=1), 16$ at King's disease stage $2(\mathrm{KDS}=2)$ and 9 at King's stage $3(\mathrm{KDS}=3)$. All participants underwent a broad battery of standardized neuropsychological tests, with published norms for the Italian population, assessing executive functions, language, memory, social cognition and visuospatial abilities (Consonni et al., 2016). The assessment of the behavioural features was performed through an interview with an adult family member or caregiver of each patient and was based on the Italian-version of the Frontal Behavioural Inventory (see Consonni, Cappa, Dalla Bella, Contarino, \& Lauria, 2019 for details). Legal copyright restrictions prevent public archiving of the neuropsychological tests and instruments. These materials can be obtained from the copyright holders in the respective cited references.

All patients with cognitive (ALSci) and/or behavioural (ALSbi) impairment as defined by recent guidelines (Strong et al., 2017) were identified. Specifically, patients with ALSci were those with either executive dysfunctions (i.e.,: impaired letter fluency or impairment on two other non-overlapping measures of executive functions which may include social cognition) or language dysfunctions or a combination of the two. ALSbi characterized ALS patients who did not meet full criteria for the frontal variant of FTD, but were rated by caregivers as having apathy or at least two non-overlapping behavioural disturbances. Patients with ALSci and/or ALSbi were subsequently assigned to the ALS impaired group (ALSimp) versus ALS-cognitively/behaviourally normal 
(ALScn) group (Table S2). The review board of the Fondazione IRCCS Istituto Neurologico Carlo Besta of Milan approved the study. Each subject was enrolled after giving written informed consent.

\subsection{Neuroimaging data analyses}

Whole-brain structural MRI scans were acquired and processed as previously described (Consonni et al., 2018). Briefly, all patients and healthy participants underwent a MRI session within a week from the neuropsychological assessment on a Philips Achieva 3.0 T. The MRI protocol included a 3D T1weighted sequence (FFE. 240 sagittal slices, $T R=9.9 \mathrm{~ms}$, $\mathrm{TE}=4.6 \mathrm{~ms}$. matrix $240 \times 240$, voxel size $=1 \times 1 \times 1 \mathrm{~mm} 3$, flip angle $=8^{\circ}$ ). Cortical reconstruction and parcellation was performed with the Freesurfer image analysis suite, documented and freely available for download online (http://surfer.nmr. mgh.harvard.edu/). The technical details of these procedures are described in prior publications (Ségonne, Pacheco, \& Fischl, 2007). Cortical thickness (CT) values were calculated in each subject (Consonni et al., 2018) and averaged on each gyral and sulcal structure of 74 bilateral regions as defined in Destrieux et al. (Destrieux, Fischl, Dale, \& Halgren, 2010). Different simple linear regression analyses were then performed in order to assess whether age (or its transformations) was effective in reducing the residual variance. Accordingly, when appropriate, the prediction equations were used to adjust CT values to remove the influence of age. Each agecorrected CT values were then transformed into $\mathrm{z}$ scores (CTz score) by subtracting the mean score of HC subjects from the individual's score and then dividing the difference by the HC group standard deviation. This transformation allowed comparing CT values of different cortical regions of different subjects with the same scale.

\subsection{Cortical thinning levels}

To obtain robust proxies of cortical involvement, five levels of cortical thinning were defined for each participant's cortical region. To this aim, regional CTz values of the HC group at the 5th, 20th, 35th and 50th percentiles were used as references. In all participants, each CTz value was converted to a new score, ranging from "0" to " 4 ", resembling cortical thinning level (Fig. 1). CTz values above the 50th percentile were transformed to the score labelled "0" (normal-like cortical thinning); CTz values at or below the 5 th percentile were transformed to "4" (pathological cortical thinning). CT values labelled "1" (35th percentile < CTz score < 50th percentile), "2" (20th percentile < CTz score $<35$ th percentile) and " 3 " (5th percentile < CTz score < 20th percentile) were intermediate score between 0 and 4 .

\subsection{Statistical analyses}

Statistical analyses were performed using SPSS (IBM SPSS statistics version 21 ), with $p$-value $<.05$ taken as significant. Demographic and clinical data were plotted and checked for normality of distribution by Kolmogorov-Smirnov tests. Parametric data (age, education, disease duration) were assessed across participant groups via one-way ANOVA

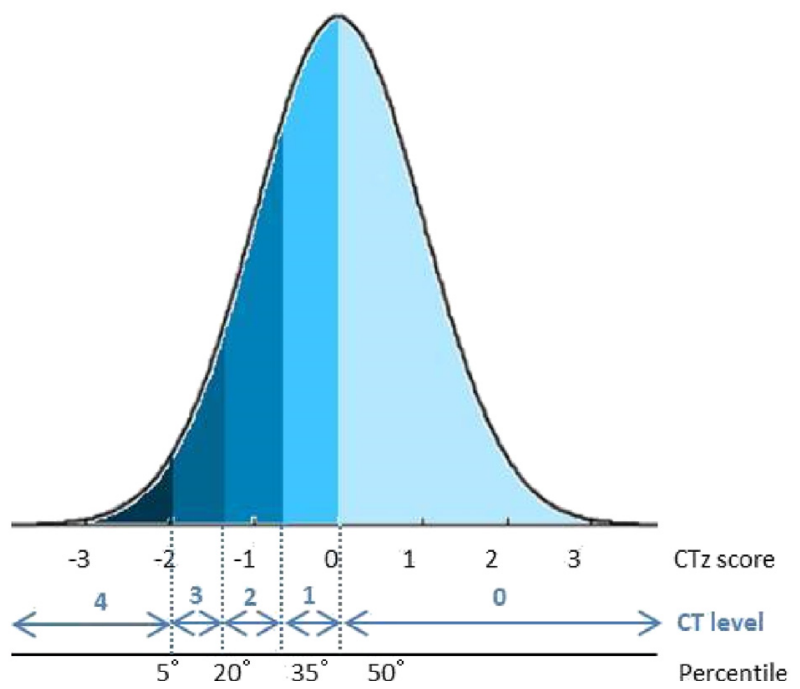

Fig. 1 - Definition of the cortical thinning levels.

followed by Bonferroni post hoc tests. Variables showing nonparametric distribution were analysed using Fisher exact test (gender, bulbar onset, bulbar symptoms, and cognitive/ behavioural impairment). The Kruskal-Wallis test was performed to test for between-group differences in ALSFRS-R scores, and Mann-Whitney $U$ tests were performed to test for specific ALS sub-group differences.

The effect of disease stages on cortical thinning levels was assessed using Jonckheere-Terpstra test. It is a nonparametric method assaying trends in data between independent ordered samples. It tests for the ordered pattern to the medians of independent groups when there is an a priori ordering of medians. We expected that our participants ordered by King's disease stage (i.e.,: $\mathrm{KDS}=0, \mathrm{KDS}=1, \mathrm{KDS}=2$ and $\mathrm{KDS}=3$, where $\mathrm{HC}$ subjects were coded as $\mathrm{KDS}=0$ ) produced a meaningful descending order of CT levels (Fig. 2A). Significant trends surviving Benjamini-Hochberg correction were highlighted. The Jonckheere-Terpstra test was also used to analyse trends in cognitive performances across disease stages. Pairwise comparisons were performed for each statistically significant trend to determine whether groups are significantly different from one another. Multiple JonckheereTerpstra test comparisons where corrected with the Benjamini-Hochberg procedure. The Jonckheere-Terpstra test was also used separately for ALSimp and ALScn groups (Table S2) to analyse group differences in cortical thinning and cognitive involvement across disease stages (Fig. 2B).

Partial correlations (corrected for age and education) were used to determine the association between cortical thinning levels, severity of clinical metrics (disease duration, ALSFRSscore) and neuropsychological indices reaching $\mathrm{p}<.05$ at the Jonckheere-Terpstra test. Correlations were bias corrected, and accelerated bootstrap 95\% intervals (BCa) were computed with 1000 bootstrap equally-sized samples obtained (Field, 2013). Only partial correlations with $\mathrm{p}<.05$ and BCa not crossing "0" were reported.

We report how we determined our sample size, all data exclusions, all inclusion/exclusion criteria, whether 
A. Jonckheere-Terpstra test (4 levels).

All patients with ALS

$0 \rightarrow \mathrm{HC}(\mathrm{N}=26)$
$1 \rightarrow$ ALS at King's stage $1(N=11)$
$2 \rightarrow$ ALS at King's stage $2(N=16)$
$3 \rightarrow$ ALS at King's stage $3(N=9)$

B1. Jonckheere-Terpstra test (4 levels).

ALSen group

$0 \rightarrow \mathrm{HC}(\mathrm{N}=26)$

$1 \rightarrow$ ALSen at King's stage $1(\mathrm{~N}=6)$

$2 \rightarrow$ ALScn at King's stage $2(N=9)$

$3 \rightarrow$ ALScn at King's stage $3(N=4)$
B2. Jonckheere-Terpstra test (4 levels).

ALSimp group

$0 \rightarrow \mathrm{HC}(\mathrm{N}=26)$

$1 \rightarrow$ ALSimp at King's stage $1(N=5)$

$2 \rightarrow$ ALSimp at King's stage $2(N=7)$

$3 \rightarrow$ ALSimp at King's stage $3(N=5)$

Fig. 2 - Steps of the Jonckheere-Terpstra test to address significant trends in cortical involvement across disease stages measured with the King staging system.

inclusion/exclusion criteria were established prior to data analysis, all manipulations, and all measures in the study. The conditions of our ethics approval do not permit public archiving of individual anonymised data. Readers seeking access to the data should contact the corresponding author. Access will be granted to named individuals in accordance with ethical procedures governing the reuse of sensitive data. Specifically, to obtain the data, requestors must complete a formal data sharing agreement.

\section{Results}

\subsection{Clinical data}

Demographic, clinical and neuropsychological data of HC and ALS patients are presented in Table 1. No significant differences were observed between HC and ALS patients for demographic variables. ALS patients subgrouped by disease clinical stage had comparable clinical features, whereas, as expected, functional disabilities measured with the ALSFRS-R scale differed between groups $(X=7.177, p=.027)$. ALSFRS- $R$ scores were higher in patients with $\mathrm{KDS} \geq 2$ compared to those with KDS = 1 (Table 1). The Jonckheere-Terpstra test explored whether cognitive and behavioural performances differed across disease stages showed different trends in neuropsychological findings between ALSimp and ALScn patients. Throughout disease stages, ALSimp showed worsening of median cognitive performance, specifically with significant reduction of phonemic verbal fluency index, language, social cognition and verbal episodic memory (Table 1). Conversely, ALScn patients showed a trend toward impairment only in global cognitive efficiency measured with the Mini Mental State Examination. Patterns of cognitive deficit across disease stages, involving multiple cognitive domains, emerged only for ALSimp patients (Table S2).

\subsection{Cortical thinning}

We found cortical thinning in motor and extra-motor brain regions. The right middle frontal sulcus and the right middleposterior cingulate gyrus survived correction for multiple comparison (Table 2). Significant trends (not surviving correction for multiple testing) toward cortical atrophy was found also in bilateral frontal and cingular cortex, left superior temporal gyrus and right superior occipital gyrus (Table 2, Fig. 3). Correlation analyses revealed that cortical thinning of the right fronto-temporal-insular cortex was specifically related to defective cognitive performances rather than disease duration or ALSFRS-R scores (Table 3). The JonckheereTerpstra test performed separately for ALScn and ALSimp patients revealed different patterns of neurodegeneration (Fig. 3, Table 4). Throughout disease stages, the ALScn group showed reduced $\mathrm{CT}$ in bilateral paracentral lobule, right frontal middle sulcus, bilateral cingular cortex, left lateral sulcus, bilateral parietal sulci and right superior occipital sulci. Conversely, ALSimp patients had specific thinning in the right hemisphere encompassing the frontal middle sulcus, the inferior temporal sulcus, the lateral sulcus and the anterior cingular cortex. Unexpectedly, we found that the horizontal ramus of the left lateral sulcus had a trend (surviving correction for multiple testing) toward increased CT between King's stages (Table 2), this trend was confirmed only in the ALScn group (Table 4). 
Table 1 - Data of Healthy controls (HC) and patients with ALS subdivided according to the King's disease stages (KDS).

\begin{tabular}{|c|c|c|c|c|c|}
\hline \multirow[t]{2}{*}{ King's stage } & \multirow{2}{*}{$\begin{array}{c}\mathrm{HC} \\
\mathrm{KDS}=0\end{array}$} & \multicolumn{3}{|c|}{ All ALS patients } & \multirow[t]{2}{*}{ Contrasts } \\
\hline & & $\mathrm{KDS}=1$ & $\mathrm{KDS}=2$ & $\mathrm{KDS}=3$ & \\
\hline & $\mathrm{N}=26$ & $\mathrm{~N}=11$ & $N=16$ & $\mathrm{~N}=9$ & \\
\hline Sex (male/female) & $16 / 10$ & $8 / 3$ & $7 / 9$ & $6 / 3$ & n.s. \\
\hline Age (yrs) & $56.8(9.9)$ & $58.0(11.4)$ & $58.7(9.9)$ & $63.0(11.8)$ & n.s. \\
\hline Education (yrs) & 11.9 (3.6) & $9.7(4.3)$ & $9.9(3.3)$ & $10.4(3.5)$ & n.s. \\
\hline ALSFRS score & - & $42.1(3.6)$ & $38.4(5.3)^{*}$ & $34.2(6.2)^{*}$ & $\mathrm{KDS}=1>\mathrm{KDS}=2^{*} \mathrm{KDS}=1>\mathrm{KDS}=3^{*}$ \\
\hline Disease duration (months) & - & $16.0(13.2)$ & $22.7(6.4)$ & $24.1(18.4)$ & n.s. \\
\hline Bulbar onset (Y/N) & - & $5 / 6$ & $2 / 14$ & $3 / 6$ & n.s. \\
\hline Bulbar symptoms (Y/N) & - & $5 / 6$ & $7 / 9$ & $5 / 4$ & n.s. \\
\hline UMN dominance & - & $1 / 10$ & $4 / 12$ & $4 / 5$ & n.s. \\
\hline \# ALSimp & - & $5(45 \%)$ & $7(43 \%)$ & $4(44 \%)$ & n.s. \\
\hline \# ALSci & - & $4(36 \%)$ & $5(31 \%)$ & $1(11 \%)$ & n.s. \\
\hline \# ALSbi & - & $0(0 \%)$ & $1(6 \%)$ & $2(22 \%)$ & n.s. \\
\hline \# ALScbi & - & $1(9 \%)$ & $1(6 \%)$ & $2(22 \%)$ & n.s. \\
\hline \multicolumn{5}{|c|}{ Neuropsychological performances - means \pm standard deviations (medians). } & Jonckheere-Terpstra (pairwise comparisons) \\
\hline MMSE & $29.35 \pm 9$ & $27.6(2.1)$ & $28.7(1.2)$ & $27.7(1.4)$ & $\mathrm{az}=-3.090, \mathrm{p}=.002\left(\mathrm{KDS}=1<\mathrm{HC}^{*} ; \mathrm{KDS}=3<\mathrm{HC}^{* * *}\right)$ \\
\hline Verbal fluency index & $3.9 \pm 1.4(3.6)$ & $6.2 \pm 4.7(4.5)$ & $6.4 \pm 4.2(4.9)$ & $5.6 \pm 2.4(5.8)$ & $\mathrm{az}=-2.450, \mathrm{p}=.014$ (n.s.) \\
\hline Digit back & $4.6 \pm 1.2(4.5)$ & $4.3 \pm 1.2(4.0)$ & $4.3 \pm 1.6(4.0)$ & $3.7 \pm 1.3(4.0)$ & n.s. \\
\hline Digit span & $6.1 \pm 1.3(6.0)$ & $5.3 \pm 1.7(5.0)$ & $5.5 \pm 1.2(6.0)$ & $5.4 \pm 1.3(6.0)$ & n.s. \\
\hline Stroop test & $18.1 \pm 7.0(17.6)$ & $23.5 \pm 12.8(20.0)$ & $24.8 \pm 13.0(21.6)$ & $26.6 \pm 1.3(18.5)$ & n.s. \\
\hline Brixton spatial anticipation & $19.1 \pm 7.3(18.0)$ & $21.5 \pm 8.2(21.0)$ & $20.8 \pm 8.3(18.5)$ & $23.3 \pm 10.5(26.0)$ & n.s. \\
\hline Object naming & $28.5 \pm 1.5(29.0)$ & $26.7 \pm 4.0(27.0)$ & $27.4 \pm 1.9(28.0)$ & $27.4 \pm 1.6(27.0)$ & $\mathrm{az}=2.469, \mathrm{p}=.014$ (n.s.) \\
\hline Auditory Comprehension & $13.9 \pm .4(14.0)$ & $13.8 \pm .4(14.0)$ & $13.5 \pm .8(14.0)$ & $13.6 \pm .7(14.0)$ & ${ }^{\wedge} \mathrm{z}=2.178, \mathrm{p}=.029$ (n.s.) \\
\hline RAVLT delayed recall & $10.5 \pm 2.4(10.5)$ & $8.9 \pm 3.4(8.0)$ & $8.6 \pm 2.8(9.0)$ & $8.4 \pm 3.3(7.0)$ & $\mathrm{az}=2.361, \mathrm{p}=.018$ (n.s.) \\
\hline Recognition memory & $24.5 \pm 3.8(25.5)$ & $23.8 \pm 4.1(23.0)$ & $22.0 \pm 5.4(22.0)$ & $24.5 \pm 2.6(24.0)$ & n.s. \\
\hline Position discrimination (VOSP) & $19.6 \pm .7(20.0)$ & $19.0 \pm 1.3(20.0)$ & $20.0 \pm 0(20.0)$ & $20.0 \pm 0(20.0)$ & n.s. \\
\hline Empathy task (SET) & $15.2 \pm 2.2(16.0)$ & $13.2 \pm 3.3(12.0)$ & $13.2 \pm 2.6(13.0)$ & $11.8 \pm 5.2(13.0)$ & $\mathrm{az}=2.527, \mathrm{p}=.012$ (n.s.) \\
\hline Emotion recognition (Ekman test) & $46.2 \pm 6.1(46.0)$ & $46.0 \pm 6.4(48.0)$ & $46.5 \pm 6.5(47.0)$ & $42.5 \pm 7.2(44.5)$ & n.s. \\
\hline FBI & - & $2.5 \pm 3.4(1.0)$ & $4.0 \pm 8.1(1.0)$ & $6.1 \pm 7.8(4.0)$ & n.s. \\
\hline
\end{tabular}

Legend: ALScn = ALS patients without cognitive and/or behavioural impairment (cognitively-normal profile); ALSimp = ALS patients with cognitive and/or behavioural impairment; ALSFRS-R = ALS Functional Rating Scale Revised; BADA = Italian Battery for the Analysis of Aphasic Deficit; ENPA = Neuropsychological Examination for Aphasia; FBI = Frontal Behavioural Inventory; HC = Healthy controls; KDS = King's Staging System; RAVLT = Rey Auditory Learning test; SET = Story-based Empathy task; UMN = Upper MotorNeuron; VOSP = Visual Object and Space Perception. Statistics: $\mathrm{a}=$ Jonckheere-Terpstra test surviving correction for multiple testing; ${ }^{*}=\mathrm{p}<.05 ; * *=\mathrm{p}<.005$. 


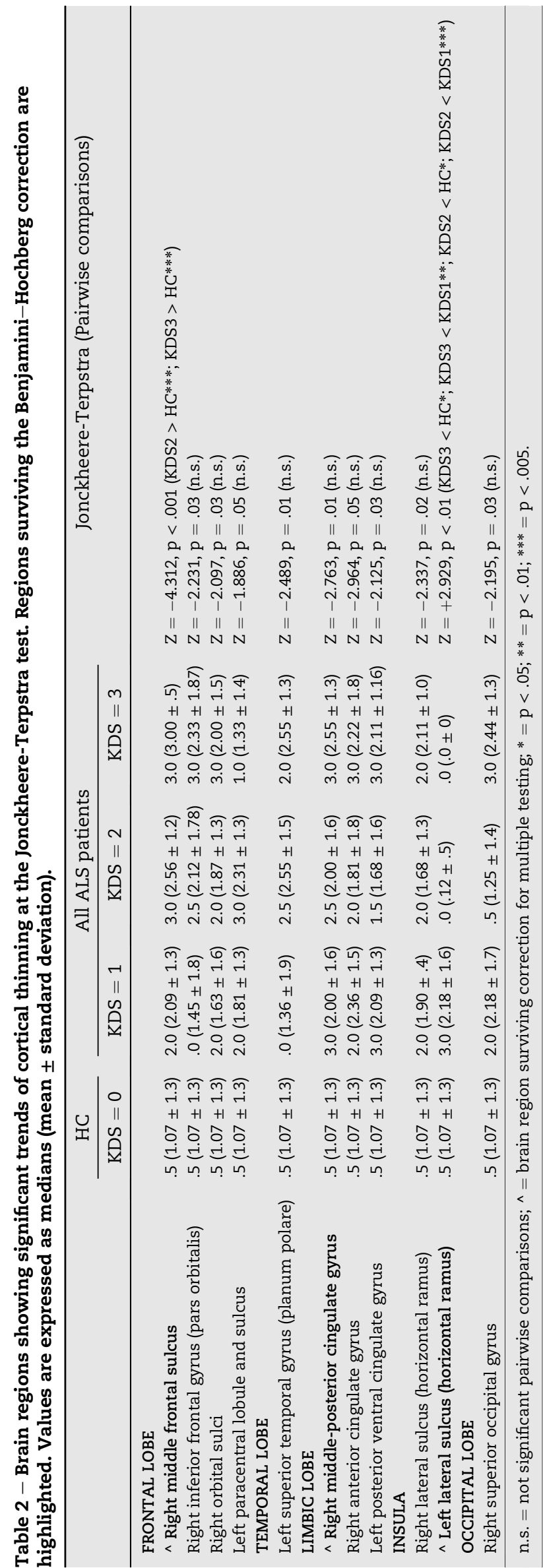

\section{Discussion}

The aim of the present study was to determine the relationship between cortical thinning and King's Clinical Staging System in a cohort of non-demented ALS patients not carrying ALS-associated gene mutations. In particular, we aimed at addressing whether cortical thinning is related to advancing disease stage, and which, if any, patterns of cortical thinning correlated to cognitive/behavioural impairment phenotype. To this aim, we divided patients according to their disease stage measured with the King's Clinical Staging System and we calculated a robust scale to be used as an approximate indicator of the level of the cortical thinning. Specifically, for each cortical region, we transformed continuous CT values into an ordinal variable (from 0 to 4), setting the extreme cut-off values of the scale above the 50th percentile (median) and over the 5th centile of the CT measure of the HC group, defining respectively the level of normal-like (CT level $=0$ ) and pathological (CT level $=4$ ) CT. Therefore, the definition of specific CT intervals, from the pathological thinning to the collapse of the best half of the measurements into a single class (Fig. 1), should limit speculations on small CT variations betweengroups.

Our findings demonstrated cortical thinning in localized motor and extra-motor brain regions across disease stages (Table 2, Fig. 3A). These encompassed the frontal regions, the left superior temporal gyrus, the bilateral cingulate cortex, the right lateral sulcus and the right superior occipital gyrus. This spreading was not related to clinical metrics (i.e., disease duration and ALSFRS-R scores) but to cognitive performances (Table 3). Indeed, reduced performances in language (naming and auditory comprehension), verbal fluency, social cognition and verbal episodic memory tasks correlated to the advanced cortical thinning of brain regions in frontotemporal and insular cortices.

Unexpectedly, we also found a trend toward thickening of the left lateral sulcus in patients at King's clinical stage 2 and 3. In a cross-sectional study, the thickening of frontal and temporal cortices in advanced ALS disease stages has been interpreted as a radiologic epiphenomenon of reactive gliosis, due to neuronal cells shrinkage and loss, which could "mask" tissue loss (Trojsi et al., 2015). In the current study, the thickening seems to be unrelated to clinical or neuropsychological data deserving future investigation with longitudinal observations. With this exception, our results seem to confirm the close relationship between poor cognitive performances and atrophy of fronto-temporal regions across disease stages. This finding is in keeping with the hypothesis that ALS patients could develop dysexecutive syndromes as a consequence of TDP-43 spreading of pathological processes, through axonal pathways, into prefrontal neocortex and further cognitive deficits when the spreading of TDP-43 reaches brain regions beyond prefrontal cortex (Brettschneider et al., 2013; Lulé et al., 2018; Schmidt, de Reus, Scholtens, van den Berg, \& van den Heuvel, 2016; Trojsi et al., 2015). Previous longitudinal studies failed to correlate cognition declines with specific cortical atrophy $(R$. A. Menke et al., 2014; Senda et al., 2011) and disease 


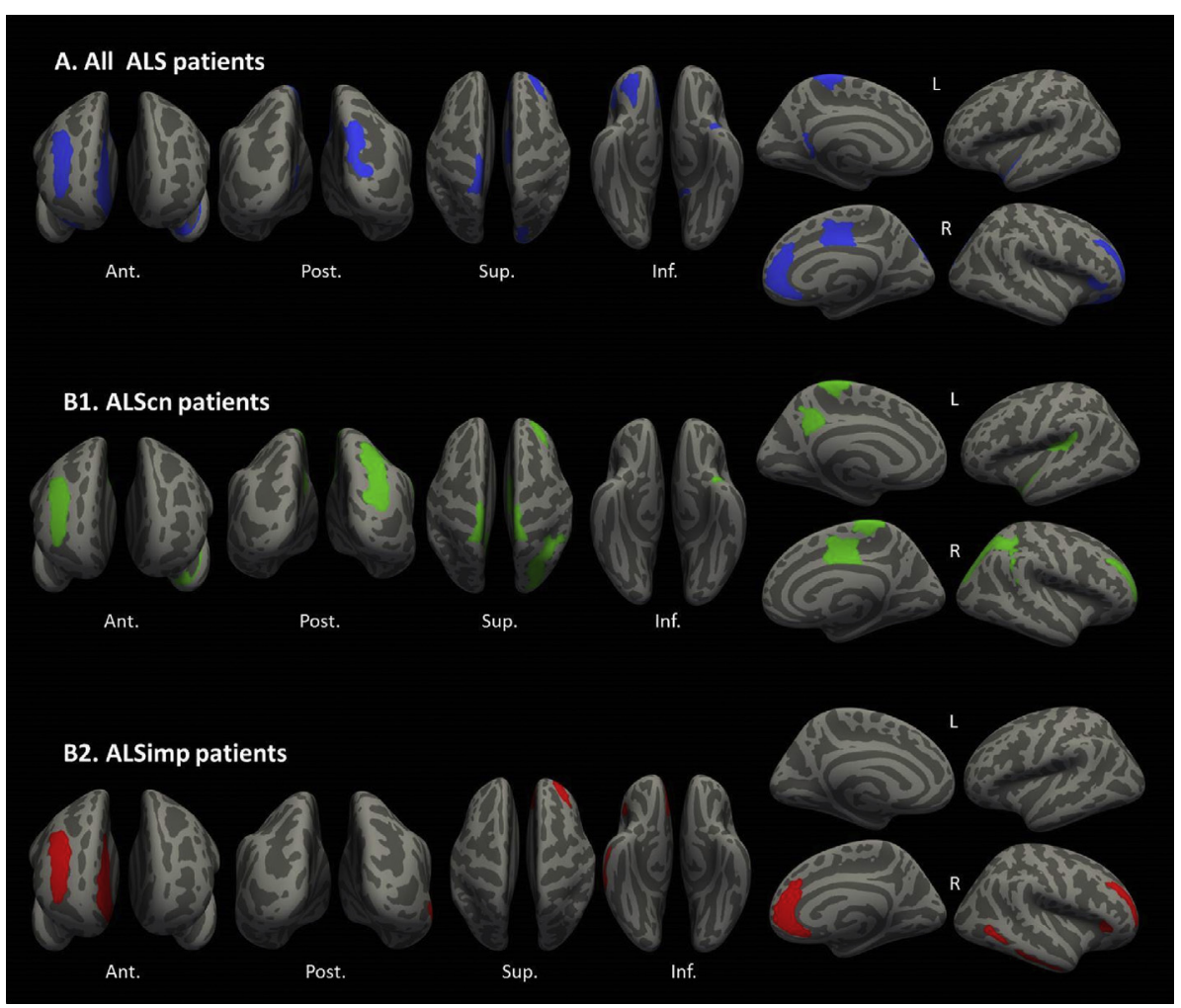

Fig. 3 - Brain regions showing significant trends toward reduce cortical thickness levels across disease stages in ALS patients (A) and in patients with cognitive-normal profile (B1, ALScn) and cognitively-behaviourally impaired patients (B2, ALSimp).

Table 3 - Significant correlations (and CIs bootstrapped intervals) between cortical thinning levels and neuropsychological performances significantly affected by advanced disease stages of ALS patients.

\begin{tabular}{|c|c|}
\hline \multicolumn{2}{|l|}{ FRONTAL LOBE } \\
\hline Right orbital sulci & Fluency index .373* (.08; .67); RAVLT -.391* (-.64; -.11) \\
\hline \multicolumn{2}{|r|}{ 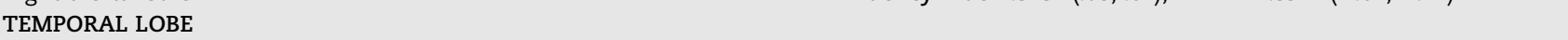 } \\
\hline Right inferior temporal sulcus & Fluency index .378* (.09; .62); RAVLT -.386* $(-.67 ;-.05)$ \\
\hline Right inferior temporal gyrus & Fluency index $.415 *(.04 ; .81)$; Comprehension $-.377 *(-.67 ; .-016)$ \\
\hline \multicolumn{2}{|l|}{ LIMBIC LOBE } \\
\hline Right anterior cingulate gyrus & RAVLT $-.429 *(-.68 ;-.09)$ \\
\hline \multicolumn{2}{|l|}{ INSULA } \\
\hline Right lateral sulcus (horizontal ramus) & Naming $-.345^{*}(-.62 ;-.17)$ \\
\hline
\end{tabular}

progression (Abrahams, Leigh, \& Goldstein, 2005; Elamin et al., 2013; Gordon et al., 2010; Schreiber et al., 2005). To our knowledge, only one longitudinal study detected localized progressive atrophy in the inferior frontal gyrus and in the dorsolateral prefrontal cortex in relation to decline in phonemic fluency performances (R. A. Menke et al., 2014). Despite several longitudinal studies documented that atrophy in ALS spreads to extra-motor regions, including frontotemporal regions, basal ganglia, thalami and cerebellum (Agosta et al., 2009; Bede \& Hardiman, 2018; R. A. Menke et al., 2014; R. A. L. Menke, Proudfoot, Talbot, \& Turner, 2018), this spreading seems most likely independent both of phenotypic presentation (Schuster et al., 2014) and motor dysfunction (Agosta et al., 2009; de Albuquerque et al., 2017; Schuster et al., 2014; Senda et al., 2011; Verstraete et al., 2012) (Table S1). The heterogeneity of ALS patient population, particularly for cognitive dysfunction, sample sizes, attrition rates and variables used to measure cognition and disease progression could have contributed in concealing the causal role of such sprouting. Therefore, to overcome the heterogeneous clinical conditions of ALS, each of which has different disease trajectories (Westeneng et al., 2018), we used the King's Clinical Staging System to measure disease burden (Balendra et al., 2014), as it is based on clinical severity rather than disease duration. The King's Clinical Staging System quantifies the spreading of motor symptoms in three different body regions involved in the disease, i.e.,: bulbar, upper limbs, and lower limbs, and on the use of non-invasive ventilation (NIV) and enteral nutrition. The number of regions involved gives the stage. Stage 4 is reached if swallowing $(4 A)$ or respiratory (4B) 
Table 4 - Brain regions of ALS subgroups showing significant trends of cortical thinning at the Jonckheere-Terpstra (J-T) test. Values are expressed as medians (mean \pm standard deviation).

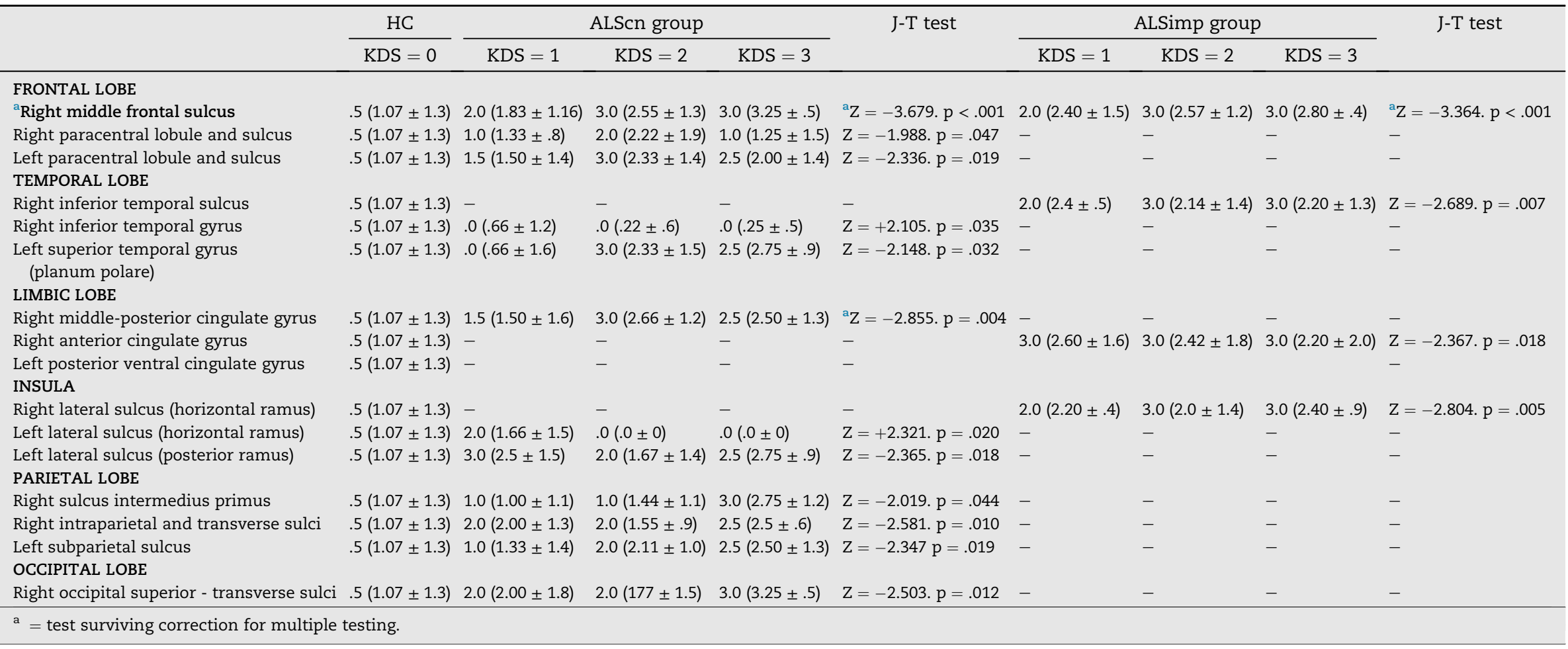


difficulty is severe enough to require intervention. None of our patients reached King's Clinical Stage 4. Given that the defining characteristics of stage 4 are nutritional deficiency requiring feeding intervention, and respiratory insufficiency, both of which impact on cognition, we use data from healthy subjects, representing King's Clinical Stage 0, to drive tests for trends in CT and cognitive performances.

The examination of the neuropsychological profiles in different King's Clinical disease stages revealed deficit in verbal fluency, empathy, language and episodic memory across disease stages. Similar to ours, other cross-sectional studies documented cognitive impairment and behavioural changes across King's clinical disease stages (Chiò et al., 2019; Crockford et al., 2018; Lulé et al., 2018; Trojsi et al., 2016). Specifically, executive dysfunction, verbal fluency and behavioural changes were significantly and negatively related to more advanced disease stage (Crockford et al., 2018), possibly confirming the hypothesis of early pathologic involvement of the prefrontal cortex in ALS (Brettschneider et al., 2013) because of their link to prefrontal cortical anomalies (Abrahams et al., 2004; R. A.; Menke et al., 2014). However, language and episodic memory have also been considered non-motor manifestations of ALS (Beeldman et al., 2016; Consonni et al., 2016; Goldstein \& Abrahams, 2013; Taylor et al., 2013), linked to brain regions extending beyond the frontal lobe (Tsermentseli, Leigh, \& Goldstein, 2012). This has led to calls for incorporating cognitive dysfunction into ALS severity scores (Al-Chalabi et al., 2016) and staging systems (Lulé et al., 2018).

Nevertheless, emerging evidence shows that cognitive impairment may also occur even from the earliest stages of ALS (Chiò et al., 2019; Crockford et al., 2018). In our sample, $43 \%$ of patients at the King's clinical stage 1 satisfied the criteria for cognitive and/or behavioural impairment. Interestingly, the frequency of ALSci, ALSbi and ALScbi, as defined by recent classification criteria (Strong et al., 2017), was similar across King's clinical stages (Table 1). This seems to contradict the hypothesis that cognitive impairment increases as disease progress, even though in a cross sectional analysis. Interestingly, the rates at which patients might develop cognitive impairment have been found to be variable in ALS (Elamin et al., 2013). With longitudinal observations, Elamin and colleagues (Elamin et al., 2013) found that, when patients with ALS were segregated into sub-cohorts based on cognitive status at baseline, a faster rate of cognitive decline was documented in the cognitively impaired subgroup, suggesting the presence of distinct subtypes. Thus, we repeated the analyses splitting patients according to their cognitive status to verify if ALScn and ALSimp patients have similar extra-motor involvement across disease stages. Results revealed that ALSimp and ALScn patients had distinct trajectories both in terms of cortical involvement and neuropsychological performances. ALScn patients had bilateral cortical involvement both in motor and extra-motor cortex with slightly reduced cognitive efficiency across disease stages (Fig. 3-B1 and Table S2), in line with the hypothesis of the TDP-43 spreading (Brettschneider et al., 2013). Moreover, we also found an unexpected trend toward cortical thickening from stage 1 to stage 3 in the left lateral sulcus of ALScn patients unrelated to any clinical features. This effect appeared to be driven by the fact that all ALScn patients, with the exception of those at King's clinical stage 1, had CT level equals to 0 , meaning healthy normal CT values (Table S2). However, we could not exclude that cortical thickening is part of a dysfunctional process (Pinto-Grau et al., 2017), an effect of cognitive reserve (Schnack et al., 2015), or a radiological epiphenomenon linked to reactive gliosis (Trojsi et al., 2015). Future longitudinal study might help to disentangle this issue.

Conversely, we showed that the neurodegenerative process in the ALSimp group predominately induced thinning of extra-motor cortices in the right hemisphere, and that thinning of the right anterior cingular cortex, lateral sulcus and inferior temporal cortex correlated with more severe disease stages (Fig. 3-B2 and Table S2). This partially confirmed others studies showing grey matter atrophy more pronounced in the right hemisphere (Bede \& Hardiman, 2018; Mezzapesa et al., 2013). The right lateralization of cortical thinning in ALS has been interpreted as an anatomical predisposition for disease related changes or a potential detection bias for cortical changes in the right hemisphere (Bede \& Hardiman, 2018). An intrinsic vulnerability of the right fronto-temporal insular cortex to cognitive impairment was confirmed also by correlation analyses revealing an association between brain regions localized in the right hemisphere with specific cognitive deficits: the thinning of the right orbital cortex with verbal fluency and episodic memory impairment, the right anterior cingulate cortex with poorer memory performances, the right inferior temporal cortex with multidomain defective performances, and the right lateral sulcus with poor naming (Table 3). Murphy et al. (Murphy et al., 2007) suggested the right hemispheric atrophy as a possible biomarker linked to behavioural abnormalities in ALS. Even though in this study we did not find behavioural changes across disease stages, previous studies reported that patients with right hemisphere anomalies exhibit disinhibited and apathetic behaviours (Consonni et al., 2019) or indifferent reactions tending to deny the extent of their disabilities (Palmieri et al., 2010). These are common profiles observed in ALS patients with behavioural disturbances (Abrahams et al., 2005; Grossman, WoolleyLevine, Bradley, \& Miller, 2007; Strong et al., 2017). From a neuropsychological perspective, we found pronounced cognitive involvement across disease stages in executive, language, memory and social cognition domains in ALSimp subgroup. Overall, our data suggest that ALSimp may have a specific pathological course distinct to that of classic ALS which is in keeping with the view that cognitive status might represent a phenotypic marker for distinct ALS subtypes (Elamin et al., 2013).

One limitation of our study is the cross-sectional design and the lack of ALS patients in the later stage of the disease, which restricted the exploration on how cognitive and behavioural symptoms could further evolve. Moreover, the small size of each subset of patients and the low statistical power of each stratified group might limit the generalizability of the results, even though the application of multiple 
comparison corrections could cover the potential significance of sub-threshold group differences.

\section{Conclusion}

Our study, using categorical cortical thinning levels (from 0 to 4) rather than row $C T$ values or zCT values, demonstrated a relationship between cognitive impairment, cortical involvement, and King's clinical disease stages, providing evidence of distinct trajectories of the spreading of degenerative processes in ALS. In particular, we showed that segregating patients according to cognitive involvement allowed finding distinct trajectories of cortical thinning with specific vulnerability of the right fronto-temporal insular cortex. This might suggest that, since the early disease stages, ALSimp patients have a specific pathological course distinct to that of the classic ALS. To our knowledge, this is the first study reporting cortical and clinical correlates across disease stages, further contributing to improve patients' deep phenotyping and paving the design of future multicentric longitudinal studies.

\section{Funding}

This work has been supported by Fondazione Regionale per la Ricerca Biomedica, Regione Lombardia [TRANS-ALS; grant number: 2015-0023].

\section{Pre-registration statement}

No part of the study procedures was pre-registered prior to the research being conducted. No part of the study analyses was pre-registered prior to the research being conducted.

\section{CRediT authorship contribution statement}

Monica Consonni: Conceptualization; Data curation; Formal analysis; Investigation; Methodology; Validation; Roles/ Writing - original draft; and Writing - review \& editing. Eleonora Dalla Bella: Project administration; Investigation; Roles/ Writing - original draft; and Writing - review \& editing. Valeria E. Contarino: Methodology; Resources; Software; Visualization; Roles/Writing - original draft; and Writing - review \& editing. Enrica Bersano: Investigation; and Writing - Review \& Editing. Giuseppe Luria: Conceptualization; Funding acquisition; Resources; Supervision; and Writing - review \& editing.

\section{Declaration of Competing Interest}

The authors have no conflict of interest to report.

\section{Acknowledgments}

We thank Drs. Patrizia Dacci, Susanna Usai, Daniele Cazzato, Ettore Salsano and Silvia Fenu of the Fondazione IRCCS
Istituto Neurologico "Carlo Besta" of Milan for referring some patients.

\section{Supplementary data}

Supplementary data to this article can be found online at https://doi.org/10.1016/j.cortex.2020.07.007.

\section{REFERENCES}

Abrahams, S., Goldstein, L. H., Simmons, A., Brammer, M., Williams, S. C., Giampietro, V., et al. (2004). Word retrieval in amyotrophic lateral sclerosis: A functional magnetic resonance imaging study. Brain: a Journal of Neurology, 127(Pt 7), 1507-1517.

Abrahams, S., Leigh, P. N., \& Goldstein, L. H. (2005). Cognitive change in ALS: A prospective study. Neurology, 64(7), 1222-1226.

Agosta, F., Gorno-Tempini, M. L., Pagani, E., Sala, S., Caputo, D., Perini, M., et al. (2009). Longitudinal assessment of grey matter contraction in amyotrophic lateral sclerosis: A tensor based morphometry study. Amyotroph Lateral Scler, 10(3), 168-174.

Al-Chalabi, A., Hardiman, O., Kiernan, M. C., Chiò, A., RixBrooks, B., \& van den Berg, L. H. (2016). Amyotrophic lateral sclerosis: Moving towards a new classification system. Lancet Neurology, 15(11), 1182-1194.

de Albuquerque, M., Branco, L. M., Rezende, T. J., de Andrade, H. M., Nucci, A., \& França, M. C. (2017). Longitudinal evaluation of cerebral and spinal cord damage in Amyotrophic Lateral Sclerosis. Neuroimage Clin, 14, 269-276.

Balendra, R., Jones, A., Jivraj, N., Knights, C., Ellis, C. M., Burman, R., et al. (2014). Estimating clinical stage of amyotrophic lateral sclerosis from the ALS Functional Rating Scale. Amyotrophic Lateral Sclerosis \& Frontotemporal Degeneration, 15(3-4), 279-284.

Bede, P., \& Hardiman, O. (2018). Longitudinal structural changes in ALS: A three time-point imaging study of white and gray matter degeneration. Amyotrophic Lateral Sclerosis \& Frontotemporal Degeneration, 19(3-4), 232-241.

Beeldman, E., Raaphorst, J., Klein Twennaar, M., de Visser, M., Schmand, B. A., \& de Haan, R. J. (2016). The cognitive profile of ALS: A systematic review and meta-analysis update. Neurologia I Neurochirurgia Polska, 87(6), 611-619.

Braak, H., Brettschneider, J., Ludolph, A. C., Lee, V. M., Trojanowski, J. Q., \& Del Tredici, K. (2013). Amyotrophic lateral sclerosis-a model of corticofugal axonal spread. Nature Reviews. Neurology, 9(12), 708-714.

Brettschneider, J., Del Tredici, K., Toledo, J. B., Robinson, J. L., Irwin, D. J., Grossman, M., et al. (2013). Stages of pTDP-43 pathology in amyotrophic lateral sclerosis. Annals of Neurology, 74(1), 20-38.

Brooks, B. R., Miller, R. G., Swash, M., Munsat, T. L., \& Diseases, W. F. o. N. R. G. o. M. N.. (2000). El escorial revisited: Revised criteria for the diagnosis of amyotrophic lateral sclerosis. Amyotroph Lateral Scler Other Motor Neuron Disord, 1(5), 293-299.

Chiò, A., Moglia, C., Canosa, A., Manera, U., Vasta, R., Brunetti, M., et al. (2019). Cognitive impairment across ALS clinical stages in a population-based cohort. Neurology, 93(10), e984-e994.

Chipika, R. H., Finegan, E., Li Hi Shing, S., Hardiman, O., \& Bede, P. (2019). Tracking a fast-moving disease: Longitudinal markers, monitoring, and clinical trial endpoints in ALS. Frontiers in Neurology, 10, 229.

Consonni, M., Cappa, S. F., Dalla Bella, E., Contarino, V. E., \& Lauria, G. (2019). Cortical correlates of behavioural change in 
amyotrophic lateral sclerosis. Neurologia I Neurochirurgia Polska, 90(4), 380-386.

Consonni, M., Catricala, E., Dalla Bella, E., Gessa, V. C., Lauria, G., \& Cappa, S. F. (2016). Beyond the consensus criteria: Multiple cognitive profiles in amyotrophic lateral sclerosis? Cortex; a Journal Devoted To the Study of the Nervous System and Behavior, 81, 162-167.

Consonni, M., Contarino, V. E., Catricalà, E., Dalla Bella, E., Pensato, V., Gellera, C., et al. (2018). Cortical markers of cognitive syndromes in amyotrophic lateral sclerosis. Neuroimage Clin, 19, 675-682.

Crockford, C., Newton, J., Lonergan, K., Chiwera, T., Booth, T., Chandran, S., et al. (2018). ALS-specific cognitive and behavior changes associated with advancing disease stage in ALS Neurology, 91(15), e1370-e1380.

Destrieux, C., Fischl, B., Dale, A., \& Halgren, E. (2010). Automatic parcellation of human cortical gyri and sulci using standard anatomical nomenclature. Neuroimage, 53(1), 1-15.

Elamin, M., Bede, P., Byrne, S., Jordan, N., Gallagher, L., Wynne, B., et al. (2013). Cognitive changes predict functional decline in ALS: A population-based longitudinal study. Neurology, 80(17), 1590-1597.

Field, A. (2013). Discovering statistics using IBM SPSS statistics (4th ed. ed.). London: SAGE Publications Ltd.

Goldstein, L. H., \& Abrahams, S. (2013). Changes in cognition and behaviour in amyotrophic lateral sclerosis: Nature of impairment and implications for assessment. Lancet Neurology, 12(4), 368-380.

Gordon, P. H., Goetz, R. R., Rabkin, J. G., Dalton, K., McElhiney, M., Hays, A. P., et al. (2010). A prospective cohort study of neuropsychological test performance in ALS. Amyotroph Lateral Scler, 11(3), 312-320.

Grossman, A. B., Woolley-Levine, S., Bradley, W. G., \& Miller, R. G. (2007). Detecting neurobehavioral changes in amyotrophic lateral sclerosis. Amyotroph Lateral Scler, 8(1), 56-61.

Lulé, D., Böhm, S., Müller, H. P., Aho-Özhan, H., Keller, J., Gorges, M., et al. (2018). Cognitive phenotypes of sequential staging in amyotrophic lateral sclerosis. Cortex; a Journal Devoted To the Study of the Nervous System and Behavior, 101, 163-171.

Menke, R. A., Körner, S., Filippini, N., Douaud, G., Knight, S., Talbot, K., et al. (2014). Widespread grey matter pathology dominates the longitudinal cerebral MRI and clinical landscape of amyotrophic lateral sclerosis. Brain: a Journal of Neurology, 137(Pt 9), 2546-2555.

Menke, R. A. L., Proudfoot, M., Talbot, K., \& Turner, M. R. (2018). The two-year progression of structural and functional cerebral MRI in amyotrophic lateral sclerosis. Neuroimage Clin, 17, 953-961.

Mezzapesa, D. M., D’Errico, E., Tortelli, R., Distaso, E., Cortese, R., Tursi, M., et al. (2013). Cortical thinning and clinical heterogeneity in amyotrophic lateral sclerosis. Plos One, 8(11), Article e80748.

Murphy, J. M., Henry, R. G., Langmore, S., Kramer, J. H., Miller, B. L., \& Lomen-Hoerth, C. (2007). Continuum of frontal lobe impairment in amyotrophic lateral sclerosis. Arch Neurol, 64(4), 530-534.

Palmieri, A., Naccarato, M., Abrahams, S., Bonato, M., D'Ascenzo, C., Balestreri, S., et al. (2010). Right hemisphere dysfunction and emotional processing in ALS: An fMRI study. Journal of Neurology, 257(12), 1970-1978.

Piao, Y. S., Wakabayashi, K., Kakita, A., Yamada, M., Hayashi, S., Morita, T., et al. (2003). Neuropathology with clinical correlations of sporadic amyotrophic lateral sclerosis: 102 autopsy cases examined between 1962 and 2000. Brain Pathology, 13(1), 10-22.

Pinto-Grau, M., Burke, T., Lonergan, K., McHugh, C., Mays, I., Madden, C., et al. (2017). Screening for cognitive dysfunction in ALS: Validation of the Edinburgh cognitive and behavioural ALS screen (ECAS) using age and education adjusted normative data. Amyotrophic Lateral Sclerosis \& Frontotemporal Degeneration, 18(1-2), 99-106.

Rascovsky, K., Hodges, J. R., Knopman, D., Mendez, M. F., Kramer, J. H., Neuhaus, J., et al. (2011). Sensitivity of revised diagnostic criteria for the behavioural variant of frontotemporal dementia. Brain: a Journal of Neurology, 134(Pt 9), 2456-2477.

Roche, J. C., Rojas-Garcia, R., Scott, K. M., Scotton, W., Ellis, C. E., Burman, R., et al. (2012). A proposed staging system for amyotrophic lateral sclerosis. Brain, 135(pt 3), 847-852.

Schmidt, R., de Reus, M. A., Scholtens, L. H., van den Berg, L. H., \& van den Heuvel, M. P. (2016). Simulating disease propagation across white matter connectome reveals anatomical substrate for neuropathology staging in amyotrophic lateral sclerosis. Neuroimage, 124(Pt A), 762-769.

Schnack, H. G., van Haren, N. E., Brouwer, R. M., Evans, A., Durston, S., Boomsma, D. I., et al. (2015). Changes in thickness and surface area of the human cortex and their relationship with intelligence. Cerebral Cortex, 25(6), 1608-1617.

Schreiber, H., Gaigalat, T., Wiedemuth-Catrinescu, U., Graf, M., Uttner, I., Muche, R., et al. (2005). Cognitive function in bulbarand spinal-onset amyotrophic lateral sclerosis. A longitudinal study in 52 patients. Journal of Neurology, 252(7), 772-781.

Schuster, C., Kasper, E., Machts, J., Bittner, D., Kaufmann, J., Benecke, R., et al. (2014). Longitudinal course of cortical thickness decline in amyotrophic lateral sclerosis. Journal of Neurology, 261(10), 1871-1880.

Ségonne, F., Pacheco, J., \& Fischl, B. (2007). Geometrically accurate topology-correction of cortical surfaces using nonseparating loops. IEEE Trans Med Imaging, 26(4), 518-529.

Senda, J., Kato, S., Kaga, T., Ito, M., Atsuta, N., Nakamura, T., et al. (2011). Progressive and widespread brain damage in ALS: MRI voxel-based morphometry and diffusion tensor imaging study. Amyotroph Lateral Scler, 12(1), 59-69.

Strong, M. J., Abrahams, S., Goldstein, L. H., Woolley, S., Mclaughlin, P., Snowden, J., et al. (2017). Amyotrophic lateral sclerosis - frontotemporal spectrum disorder (ALS-FTSD): Revised diagnostic criteria. Amyotrophic Lateral Sclerosis \& Frontotemporal Degeneration, 18(3-4), 153-174.

Taylor, L. J., Brown, R. G., Tsermentseli, S., Al-Chalabi, A., Shaw, C. E. Ellis, C. M., et al. (2013). Is language impairment more common than executive dysfunction in amyotrophic lateral sclerosis? Neurologia I Neurochirurgia Polska, 84(5), 494-498.

Trojsi, F., Caiazzo, G., Corbo, D., Piccirillo, G., Cristillo, V., Femiano, C., et al. (2015). Microstructural changes across different clinical milestones of disease in amyotrophic lateral sclerosis. Plos One, 10(3), Article e0119045.

Trojsi, F., Santangelo, G., Caiazzo, G., Siciliano, M., Ferrantino, T. Piccirillo, G., et al. (2016). Neuropsychological assessment in different King's clinical stages of amyotrophic lateral sclerosis. Amyotrophic Lateral Sclerosis \& Frontotemporal Degeneration, 17(3-4), 228-235.

Tsermentseli, S., Leigh, P. N., \& Goldstein, L. H. (2012). The anatomy of cognitive impairment in amyotrophic lateral sclerosis: More than frontal lobe dysfunction. Cortex; a Journal Devoted To the Study of the Nervous System and Behavior, 48(2), 166-182.

van Es, M. A., Hardiman, O., Chio, A., Al-Chalabi, A., Pasterkamp, R. J., Veldink, J. H., et al. (2017). Amyotrophic lateral sclerosis. Lancet, 390(10107), 2084-2098.

Verstraete, E., Veldink, J. H., Hendrikse, J., Schelhaas, H. J., van den Heuvel, M. P., \& van den Berg, L. H. (2012). Structural MRI reveals cortical thinning in amyotrophic lateral sclerosis. Neurologia I Neurochirurgia Polska, 83(4), 383-388.

Verstraete, E., Veldink, J. H., van den Berg, L. H., \& van den Heuvel, M. P. (2014). Structural brain network imaging shows expanding disconnection of the motor system in amyotrophic lateral sclerosis. Hum Brain Mapp, 35(4), 1351-1361.

Westeneng, H. J., Debray, T. P. A., Visser, A. E., van Eijk, R. P. A., Rooney, J. P. K., Calvo, A., et al. (2018). Prognosis for patients with amyotrophic lateral sclerosis: Development and validation of a personalised prediction model. Lancet Neurology, 17(5), 423-433. 